\title{
FURTHER STUDY OF JINR TAU-CHARM FACTORY DESIGN
}

\author{
E. Perelstein, V. Alexandrov, V. Antropov, O. Arkhipov, P. Beloshitsky, \\ L. Bobyleva, V. Kazacha, N. Kazarinov, A. Krasnykh, V. Mironov, \\ D. Kaltchev, L. Onischenko, A. Sissakian, Yu. Smirnov, Ts. Vylov \\ Joint Institute for Nuclear Research, Dubna, Russia \\ V. Belov, B. Mudiugin, A. Popov, D. Serebrennikov \\ Scientific Research Institute of Electrophysical Apparatus, St. Petersburg, Russia \\ I. Kvashonkin, E. Petrov, I. Umansky \\ Russian Institute of Powerful Radioconstruction, St. Petersburg, Russia
}

\section{INTRODUCTION}

At present there are several proposals of the magnet lattices suitable the standard and monochromatization regimes for tan-charm factory [?], [?],[?]. In this paper the main features of the design using versatile lattice are discussed. The beam and machine parameters and a short description of some factory systems are presented to illustrate the design feasibility.

\section{Magnet lattice of TCF}

Now we have changed magnet lattice of tan-charm collider. The previous one [?] was based on conventional flat beam gcheme. The new lattice [?] is veraatile and allows to use both standard scheme and monochromatization one. To have the possibility of a use both schemes the versatile lattice should to fulfil few conditions. Two of them are of most importance. The first is a possibility to change an emittance approximately in 20 times: from $300 \div 400$ $\mathrm{nm}$ for the conventional acheme up to $15 \div 20 \mathrm{~nm}$ for the scheme with monochromatization. The gecond is a necesaity of a change polarity in microbeta quadrupoles. The last condition is a consequence of a fact one wants to gain in energy resolntion without loss of a luminosity in the case the monochromatization is made in the vertical plane.

The big change of an emittance is achieved by ure of different phase advances in a regular cell for conventional scheme and monochromatization scheme and by appropriate use of wigglers. In high emittance lattice (conventional scheme) $60^{\circ}$ phase advance is used in a regular cell. Two variants of wigglers switching to incresse an emittance compared with those generated in bending magnets are now under consideration. In the first variant Robinson wigglers reduce horizontal damping partition number $J_{x}$ from 1 to 0.6. Robinson wiggler consists of 4 blocks each of $0.23 \mathrm{~m}$ long. It is necessary 4 such wigglers located close to each of 4 dispersion suppreseors with gradient $G=4.3 \mathrm{~T} / \mathrm{m}$ and magnetic field $B=0.35 T$. Four dipole wigglers each of $1.0 \mathrm{~m}$ long with magnetic field $B=1.9 \mathrm{~T}$, located in first half cell of suppressor, produce an additional increase of emittance. The magnetic elementa location and lattice functions in this variant are shown in Fig. 1. In the second variant the dipole wigglers only are used to increase an emittance. The magnetic field in dipole wigglers is $2.6 \mathrm{~T}$ in this case.

When comparing two variants (Table 1) one sees the first one is preferable from the point of view smaller RF voltage is needed to keep bunches short. On the other hand, in the second variant damping times are amaller that is important for the injection and beam-beam effects. The final choice can be done after comprehensive stady problems mentioned above and others such as multibunch instabilities, broadband impedance restriction etc.

For monochromatization scheme, the horizontal phase advance is $90^{\circ}$ in a regular cell. Dipole wigglers are switched off. Robinson wigglers are switched on in a way to reduce an emittance by increasing horizontal damping partition number $J_{x}$ from 1 to 2 . The value of gradient in wiggler is $\mathrm{G}=7.3 \mathrm{~T} / \mathrm{m}$ and magnetic field $\mathrm{B}=1.9 \mathrm{~T}$. The dispersion suppressor is made flexible enough to cancel dispersion in both $60^{\circ}$ lattice and $90^{\circ}$ one.

To make a small beta's at interaction point (I.P.) $\beta_{x}^{\star}=$ $0.30 \mathrm{~m}$ and $\beta_{y}^{*}=0.01 \mathrm{~m}$ two quadrupoles are used instead of triplet [?]. When changing polarities in quads for monochromator optics (Fig. 2), the values of beta's become $\beta_{x}^{*}=0.01 \mathrm{~m}, \beta_{y}^{*}=0.15 \mathrm{~m}$ and vertical dispersion $D_{y}^{\star}=0.36 \mathrm{~m}$. The preliminary vertical separation is made by vertical separator. The vertical distance between beam axis in parasitic I.P. is $24 \sigma_{y}$ for conventional scheme and $11 \sigma_{y}$ for monochromatization scheme.

The chromaticity correction is made now for high emittance lattice. With $60^{\circ}$ phase advance per regular cell 6 sextupole families have been used to correct chromatic properties. The solution have been found provides $\pm 1.8 \%$ of energy acceptance. The beam lifetime for conventional scheme is defined by beam-beam brearnstrahlung predominantly and, to some extent, by neutral gas scattering. With longitudinal acceptance $1.8 \%$ and average pressure in vacuum chamber of $2 \cdot 10^{-7} \mathrm{~Pa}$ it is of 5 hours. The beam lifetime for monochromatization scheme is defined by Touschek effect and depends strongly on dynamic aperture. Its estimate gives $1 \div 3$ hours [?], [?]. The main parameters of ter-charrn collider are presented in Tuble 1. 


\begin{tabular}{|c|c|c|c|c|}
\hline & & $\begin{array}{c}\text { Monochrom. } \\
\text { scheme }\end{array}$ & $\begin{array}{c}\text { Standard } \\
\text { Var.1 }\end{array}$ & $\begin{array}{l}\text { scheme } \\
\text { Var.2 }\end{array}$ \\
\hline Energy, GeV & $\mathbf{E}$ & 2.0 & 2.0 & 2.0 \\
\hline Luminosity, $\mathrm{cm}^{-2} \mathrm{sec}^{-1}$ & $\mathbf{L}$ & $8.0 \cdot 10^{32}$ & $9.2 \cdot 10^{32}$ & $9.4 \cdot 10^{32}$ \\
\hline C.M. energy resolution, $\mathrm{MeV}$ & $\sigma_{w}$ & 0.14 & 1.8 & 2.4 \\
\hline Circumference, $\mathrm{m}$ & $\mathrm{C}$ & 378 & 378 & 378 \\
\hline Natural emittance, nm & $\epsilon_{0}$ & 15.1 & 388 & 393 \\
\hline Bending radius in arc, $m$ & $\rho$ & 11.5 & 11.5 & 11.5 \\
\hline Damping times, msec & $\tau_{s} / \tau_{y} / \tau_{s}$ & $1 \theta / 3 \theta / 3 \theta$ & $43 / 25 / 11$ & $18 / 18 / 8.7$ \\
\hline Momentum compaction & $\alpha$ & $7.85 \cdot 10^{-3}$ & $1.63 \cdot 10^{-2}$ & $1.63 \cdot 10^{-2}$ \\
\hline Energy spread & $\sigma_{E}$ & $7.18 \cdot 10^{-4}$ & $6.23 \cdot 10^{-4}$ & $8.50 \cdot 10^{-1}$ \\
\hline Total current, $\mathrm{mA}$ & I & 441 & 516 & 536 \\
\hline Number of bunches & $\mathbf{k}_{\mathbf{b}}$ & 30 & 30 & 30 \\
\hline RF voltage, MV & V & $\mathbf{5}$ & 10 & 16 \\
\hline RF frequency, $\mathbf{M H z}$ & $\mathrm{f}_{R F}$ & 476 & 476 & 476 \\
\hline Harmonic number & $\mathrm{q}$ & 600 & 600 & 600 \\
\hline Energy losses per turn, kV & $U_{0}$ & 131 & 200 & 262 \\
\hline Bunch length, mm & $\sigma_{s}$ & 7.83 & 6.93 & 7.47 \\
\hline Longitudinal impedance, $\mathrm{Ohm}$ & $\left|Z_{n} / n\right|$ & 0.18 & 0.21 & 0.42 \\
\hline Beta functions at L.P., m & $\beta_{z}^{*} / \beta_{y}^{*}$ & $0.01 / 0.15$ & $0.30 / 0.01$ & $0.30 / 0.01$ \\
\hline Vertical dispersion at I.P., $\mathrm{m}$ & 0.36 & 0. & 0. & \\
\hline Beam-bearn parameters & $\xi_{x} / \xi_{y}$ & $0.040 / 0.029$ & $0.04 / 0.04$ & $0.04 / 0.04$ \\
\hline
\end{tabular}

Table 1: List of parameters of tan-charm collider

\begin{tabular}{|c|c|c|c|c|c|c|}
\hline & N & AC & DC & $\mathbf{R}$ & L & Power \\
& & A & A & $\mathrm{m} \Omega$ & $\mathrm{mH}$ & $\mathbf{k W}$ \\
\hline Dipoles & $\mathbf{4 8}$ & $\mathbf{5 8 5}$ & $\mathbf{8 6 5}$ & 11 & 6.0 & 1700 \\
Quads I & $\mathbf{3 6}$ & $\mathbf{5 6 5}$ & $\mathbf{8 6 5}$ & 10 & 1.8 & 1200 \\
Quads II & $\mathbf{3 6}$ & $\mathbf{5 6 5}$ & $\mathbf{8 6 5}$ & $\mathbf{3}$ & 0.23 & $\mathbf{3 0 0}$ \\
Sext. I & $\mathbf{3 0}$ & $\mathbf{8 6}$ & 140 & $\mathbf{8 . 4}$ & 1.6 & 30 \\
Sext. II & $\mathbf{3 0}$ & 115 & 185 & $\mathbf{8 . 4}$ & 1.6 & 40 \\
\hline
\end{tabular}

Table 2: Booster Power Supply Dates

\section{MAGNET SYSTEM}

According to the factory cyclogram [?] the booster repetition rate is $25 \mathrm{~Hz}$. The ceramic booster vacuum chamber is designed here and the white-circuit type of the resonant scheme of booster power supply is adopted. The compensation of the pulse loss is realized by the isolation reactors from the special pulse power supplies. The design dates of the booster power supply are presented in Table 2 .

There are a three snbsystems for the system of the power supply of the tau-charm factory storage ring. They feed: 1) superconducting quadrupoles and dypole wigglers; 2) septum magnets; 3) dipoles, quadrupoles, sextupoles, Robinson wigglers. The third group has a big energy capacitance and is quit expensive. It consists of 160 dipole magnets, 8 vertical bend magnets, 16 wigglers, 234 quads and 112 arc sextupoles. There are a 48 group for the power supply to this system. Each chain has got a separate power source. The prototype of the power source is DC Sources that have been designed at Institute of Electrophysical Apparatus (St.Petersburg) and "Electrotechnic" firm (Tallin, Estonia). The parameters of this power sources allow to get the driving range $(0.6-1.0) \cdot P_{\text {nom }}$ with stability coeffcient $\pm 10^{-5}$.

The cores of the storage ring magnet is made from the laminated electrical steel (type 2212) with thickness of 1.5 $\mathrm{mm}$. The main ring dipoles have C-shape with the dimensions $440 \times 580 \mathrm{~mm}$ with the gap of $60 \mathrm{~mm}$. and will be made from laminated electrical steel (type 2411) with thickness of $0.5 \mathrm{~mm}$.

\section{VACUUM SYSTEM}

The beam particle breamstrahlung in the residual gas atmosphere contribates to the beam lifetime. For the typical residual gas composition $\left(70 \% \mathrm{H}_{2}, 20 \% \mathrm{CO}, 10 \%\right.$ $\mathrm{CO}_{2}$ ) and the pressure $2 \cdot 10^{-7} \mathrm{~Pa}$ vacuum lifetime is about $30 \mathrm{~h}$. The gas loading is defined mainly by synchrotron radiation (SR) desorbtion. The photodesorbtion coefficient is adopted to be equal to $\eta=10^{-8}[\mathrm{~mol} / \mathrm{phot}]$, that corresponds to the dose of $50 \mathrm{~A} \cdot \mathrm{h}$ [?]. Providing the chemical cleaning and heating of the vacuum chamber the outgassing rate of aluminum doesn't exceed $g=$ $10^{-9}[\mathrm{~m} \cdot \mathrm{Pa} / \mathrm{sec}]$, that much less then stimulated desorbtion. 
The vacuum chamber of tan-charm factory is manufactured from aluminum and designed in such a manner that SR goes through next straight section and is absorbed at the bending magnet end (Fig. 2). The vacuum volume at the bending magnet region is divided on two parts: the beam chamber and the antechamber. The chamber aperture is $49 \times 64 \mathrm{~mm}$ and it isn't varied along the whole chamber length. The gap between the beam chamber and antechamber has been chosen to folfil the condition of 95\% SR passing through. The SR abeorber is made as water-cooling couplers tube with extended surface. The absorber have the ontlet SR extracting windows for the user purposes.

The stimulated ontgassing per a bending magnet is equal to $8 \cdot 10^{-8}\left[\mathrm{~m}^{3} \cdot \mathrm{Pa} / \mathrm{sec}\right]$. Using the combined pumps with the pamping speed $0.4 \mathrm{~m}^{3} / \mathrm{sec}$, one gets the pressure about $2 \cdot 10^{-7} \mathrm{~Pa}$ at the abeorber location. The additional pamp is used for the pumping of the remaining part of vacunm volume and provides the pressure at the level $2 \cdot 10^{-8} \mathrm{~Pa}$.

\section{RF SYSTEM}

To compensate energy beam losses and to keep bunches short $500 \mathbf{M H z}$ superconducting RF cavities is planned to ure. The total value of SR and HOM loses at energy $E=2.0 \mathrm{GeV}$ is of order $300 \mathrm{~kW}$ and the maximum $\mathrm{RF}$ voltage is of $16 \mathrm{MV}$ for one ring. The voltage amplitude and phase tolerances are defined by beam quality demands. The fluctuations in voltage amplitude enhance beam spread. Putting the tolerable increase of beam spread $5 \%$ one gets limitation for RF voltage fiuctuations $\Delta V / V \leq 5 \cdot 10^{-3}$. The phase shift between RF modules excites the synchrotron oscillations. Putting restriction for their amplitude to be $\leq 1 \mathrm{~mm}$, one gets $|\delta \phi.| \leq 1^{\circ}$.

The RF power supply ocheme for tan-charm factory is gronded on the principle of separate supply of each cavity like [7]. The main questions are the choice of an adequate final stage amplifier and the feeder line design. Klystrons developed at "SVETLANA" (St.Petersburg) satisfied tancharm factory requirements and have the following parameters: output power - $80 \mathrm{~kW}$, frequency - $500 \mathrm{MHz}$, efficiency -0.58 , amplification $-45 \mathrm{~dB}$, collector voltage -16 $\mathrm{kV}$, collector current $-8.6 \mathrm{~A}$.

Each feeder line includes a ferrite circulator with a ballast load, that allows to refuse from phase shifter using. The effective automatic phase control is provided by the electronic phase shifter in a preliminary stage of a $\mathbf{R F}$ amplifier. The main cooxial feeder connecting a circulator output and a cavity inpat has the cross section dimensions $160 \times 70 \mathrm{~mm}$ and the wave impedance $50 \mathrm{Ohm}$. Thus the RF power supplier consists of 4 independent FR lines with the total outpat power $320 \mathrm{~kW}$.

\section{REFERENCES}

[1] A. Fans-Golfe and J. Le Duff "A Versatile Lattice for a Tan-Charm Factory That Inclades Monochromati- zation Scheme" (LAL-RT/92-01, Orsay, 1992)

[2] Alex\&nder Zholents "Polarized $\mathrm{J} / \psi$ Mesons at a Tau-Charm Factory with a Monochromator Scheme" (CERN SL/92-27(AP), 1992)

[3] Paul Beloshitsky "A Magnet Lattice for a Tau-charm Factory Suitable for Both Standard Scheme and Monochromatization Scheme" (LAL-RT/ 92-09, Orsay, 1992)

[4] E. A. Perelstein e.a. "JINR Tau-Charm Factory Study" (Proceeding of XV International Conference on High Energy Accelerators, Hamburg,1992)

[5] M. V. Danilov e.a. "Conceptual Design of TauCharm Factory in ITEP" (Proceeding of XV International Conference on High Energy Accelerators, Hamburg, 1992)

[6] M. Bernardini "Vacuum System for ELETTRA of Synchrotron Trieste" (Proceedings of the SR Vacuum Workshop, Wako-shi, Saitama, Japan, 1992, p.272)

[7] B. Barish e.a. "Tau-Charm Factory Design" (SLACPUB-5180, Stanford, 1990)

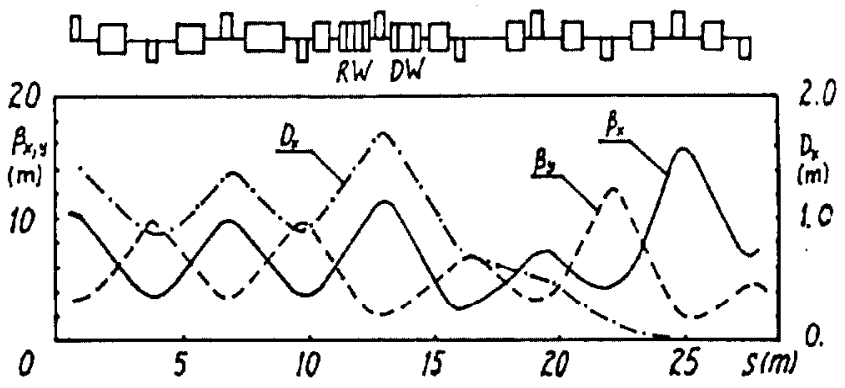

Fig. 1. Lattice functions in regular cell and dispersion suppressor.

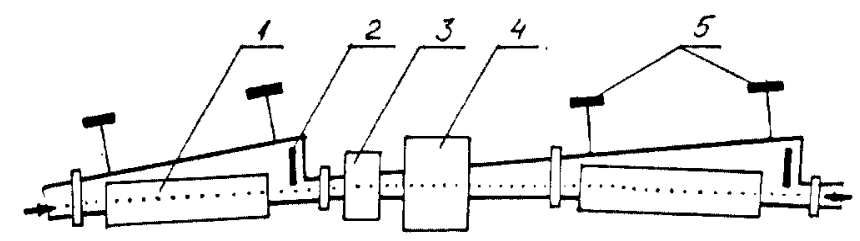

Fig. 2. Periodic cell scheme (1-dipole, 2-SR absorber, 3-sextupole, 4-quadrupole, 5-pumps). 